\title{
La insoportable levedad de la política nacional de salud mental para Colombia: reflexiones a propósito de la propuesta de ajuste en $2014^{*}$
}

\author{
The Unbearable Lightness of the National Mental Health Policy in Colombia: Reflections on the 2014 \\ Amendment Proposal
}

A insustentável leveza da politica nacional de saúde mental para a Colômbia : reflexões a propósito da proposta de ajuste em 2014

\author{
Carlos José Parales-Quenza ${ }^{\text {a }}$ \\ Universidad Nacional de Colombia, Colombia \\ ORCID: http://orcid.org/0000-0003-3239-3606 \\ Zulma Consuelo Urrego-Mendoza \\ Universidad Nacional de Colombia, Colombia \\ ORCID: http://orcid.org/0000-0003-1732-4725 \\ Edwin Herazo-Acevedo \\ Universidad Antonio Nariño, Colombia \\ ORCID: http://orcid.org/0000-0002-9461-7997
}

DOI: https://doi.org/10.11144/Javeriana.rgps17-34.ilpn

\section{Resumen:}

La salud mental es una problemática de interés para muchos gobiernos. En los últimos veinte años, en Colombia se han hecho varios intentos de formular una política nacional, cuya recepción por la sociedad y sus instituciones ha sido fútil. Desde la perspectiva del equipo consultor para el ajuste de la Política Nacional de Salud Mental Colombiana en 2014, se elaboró un recuento de tales iniciativas desde 1994 hasta 2015, con énfasis en lo sucedido en 2014, y se analizaron diversos antecedentes que considerar para comprender las dificultades en la implementación de una política pública al respecto. Entre las dificultades afrontadas se encontraron la diversidad de conceptualizaciones sobre salud mental, la falta de voluntad política, las características propias de los sistemas de salud y el estigma social que rodea a los padecimientos mentales. Tales aspectos, entre otros, han hecho ardua y compleja la tarea de formular e implementar políticas nacionales de salud mental.

Palabras clave: política de salud, salud mental, salud pública, políticas públicas, formulación de políticas, Colombia.

\section{Abstract:}

Despite good intentions, governments have found difficult to address the mental health. There have been several attempts in the last twenty years to formulate a national mental health policy in Colombia, all of which have found resistance on the part of society and institutions. We offer a recount of the multiple attempts to develop and implement a national policy of mental health, and discuss possible factors behind the failure to achieve a national mental health policy. Among the factors that account for the difficulty in formulating and implementing mental health policies are competing conceptualizations of mental health, barriers related to the characteristics of the health system, lack of political will, and the social stigma and discrimination experienced by people living with mental health problems.

Keywords: Health policy, mental health, public health, public policies, policy making, Colombia.

Resumo:

A saúde mental é uma problemática de interesse para muitos governos. Nos últimos vinte anos, na Colômbia tem se feito varias tentativas de formular uma política nacional, cuja recepção pela sociedade e suas instituições foi fútil. Da perspectiva da equipe consultiva para as alterações da Política Nacional de Saúde Mental Colombiana em 2014, foi elaborada uma recontagem de tais iniciativas desde 1994 até 2015, com ênfase no acontecido em 2014, e analisaram-se diversos antecedentes a considerarem para compreender as dificuldades na implementação de uma política pública nesse sentido. Entre as dificuldades afrontadas encontraram-se a diversidade de conceptualizações sobre saúde mental, falta de vontade política, as características próprias dos

a Autor de correspondencia. Correo electrónico: cparalesq@unal.edu.co 
sistemas de saúde e o estigma social em torno das doenças mentais. Tais aspectos, dentre outros, tornam árdua e complexa a tarefa de formular e implementar políticas nacionais de saúde mental.

Palavras-chave: política de saúde, saúde mental, saúde pública, políticas públicas, formulação de políticas, Colômbia.

\section{Introducción}

La salud mental se reconoce generalmente como prioridad, relacionada con planes globales de desarrollo socioeconómico [1], y el trabajo en su promoción y mantenimiento constituye un reto para los gobiernos del mundo [2]. El Plan de Acción Integral de Salud Mental para 2013-2020 establece una guía y compromiso para aquellos Estados miembros de la Organización Mundial de la Salud (OMS) en favor de la salud mental [2], incluida Colombia, que hace parte de dicha organización desde 1959 [3]. No obstante, cada Estado miembro debe ajustar tales lineamientos generales a sus características particulares, y surgen allí discusiones sobre las formas efectivas de abordarlas. Asumir una agenda política internacional en el plano de lo nacional, implica recorrer cuatro etapas sucesivas, correspondientes a grupos de tareas relacionadas con los distintos momentos del ciclo de la política pública: asumir la agenda, implementarla, hacerle seguimiento y evaluarla [4].

Cada país debe interpretar el marco normativo general de la agenda internacional a partir de sus propios valores, encarnados en los actores sociales ejecutores y destinatarios involucrados en el proceso; ello da lugar a una multitud de interpretaciones y reinterpretaciones, no siempre concordantes, dependientes de dichos actores, de su número y variedad, del nivel de abstracción o concreción de la agenda inicial, de la capacidad de negociación entre actores sociales y de la estabilidad o inestabilidad del contexto en que se va a llevar a cabo la implementación. Si todo ello trascurre de modo favorable, ocurrirá un conjunto de adaptaciones y contextualizaciones sucesivas que desembocarán en una política nacional finalmente trazada y ejecutada efectivamente dentro de un contexto nacional concreto [5].

La formulación de una política pública de salud mental apropiada para las realidades de nuestro país no ha sido la excepción en ese marco. Como aporte para la comprensión y posible superación de las dificultades al respecto, este ensayo propone reflexionar, desde la perspectiva del equipo consultor a cargo, sobre la experiencia de actualización de la política pública en salud mental colombiana llevada a cabo en 2014. La reflexión se realiza a la luz de experiencias que le antecedieron y siguieron, con el fin de identificar controversias y obstáculos de diversa índole que pudieran estar afectando la posibilidad de lograr que en Colombia se consiga la formulación e implementación efectiva de una política pública nacional de salud mental.

\section{Método}

Se realiza una aproximación sucesiva a las particularidades colombianas en torno al objeto de discusión señalado, a partir de premisas generales sobre el tema. En primer término, se presenta un balance sobre las controversias y obstáculos que a lo largo del mundo han impactado los ejercicios de construcción de políticas públicas en salud mental; luego se esboza un breve recuento sobre los antecedentes colombianos en el campo abordado, hasta el año 1993 en que se realiza el llamado Primer Estudio Nacional de Salud Mental. Se continúa con lo sucedido en el país al respecto durante el periodo 1994-2013, año de formulación de la Ley 1616 de salud mental, y se culmina la exposición con lo acontecido luego de dicha norma, bajo la cual fue realizado el ejercicio de actualización de la Política Nacional de Salud Mental de 2014, que es foco esencial de este escrito. Se incluyen además algunas reflexiones sobre lo ocurrido en los dos años posteriores a dicha actualización. El ensayo finaliza con la discusión de nuestras reflexiones a la luz de otras experiencias publicadas en el tema, junto con las respectivas conclusiones. 


\section{Resultados}

\section{Controversias y obstáculos en torno a la formulación de políticas de salud mental en el mundo}

La salud es simultáneamente un tema clínico y social; por definición, salud mental no es la ausencia de enfermedad mental [2]. No obstante, la ampliación del concepto complica la formulación e implementación de políticas públicas, que de todas formas precisan de la definición de un foco, debido al número y diversidad de actores que involucran. Al mismo tiempo, trastornos mentales y condiciones sociales se encuentran fuertemente imbricados, como lo han señalado y sustentado diversos autores tanto desde el campo de la salud mental como desde el de la salud pública, por lo cual su escisión para el análisis resulta artificial.

Sobre las relaciones entre pobreza y salud mental, por ejemplo, se sabe que los pobres son más vulnerables, tienen más dificultades en el acceso a la atención, y que la pobreza está relacionada con otras variables sociales muy importantes para la salud mental como lo son el empleo y la educación [6]. En la práctica, sin embargo, la separación entre sociedad y salud mental individual tiene lugar y ello repercute en el trazado de intervenciones efectivas para impactar la segunda. En especial, es preciso considerar el contexto de un predominio biomédico dentro del pensamiento científico en torno a la salud mental, desde la primera mitad del siglo XX [7] hasta nuestros días [8].

En ese marco, se arguye falta de referentes empíricos concretos que permitan corroborar la efectividad de las intervenciones, debido en parte a la complejidad del fenómeno y en virtud de los factores que intervienen, pero en general pareciera que la solución que se ha encontrado es la de asumirla como un fenómeno invisible. En todo caso, ignorar una problemática es la peor opción.

Múltiples barreras de acceso, que en nuestro país datan incluso de tiempos coloniales, aunque reactualizados periódicamente a la luz del contexto cambiante de la atención hasta nuestros días [9,10,11], dejan sin tratamiento a la mayoría de las personas con problemas de salud mental y trastornos mentales. A ello se suma el problema de los costos; los sistemas de salud son renuentes al aseguramiento de una cobertura que demanda importantes recursos y cuyos resultados no pueden observarse directamente.

Se encuentra además la complicación de la definición: ¿qué es salud mental?, ¿se trata de la ausencia de trastornos mentales clínicamente diagnosticables o de la calidad del tipo de relaciones que las personas establecen entre sí y con el mundo en el que habitan? Si se acoge una definición del tipo de la primera perspectiva, entonces las líneas de acción suponen disponer de recursos sectoriales suficientes para brindar oportunidad y calidad en el cuidado, lo que exige asumir costos que los sistemas de salud alegan no tener capacidad de cubrir; sin embargo, en la lógica de la racionalidad económica, si el funcionamiento de un sistema no le diera utilidad a ningún actor, se reformaría.

La segunda perspectiva es intersectorial, enfatiza la promoción y prevención e implica el riesgo de volver el concepto demasiado versátil y volátil; la difusión de la conceptualización conlleva el problema de la difusión de responsabilidades. La intersectorialidad plantea problemas de coordinación que dificultan el logro de objetivos de intervención integral en fenómenos complejos y multidimensionales. La consecuencia es la formulación e implementación de políticas sociales no integradas.

Las implicaciones de la conceptualización son profundas. Los indicadores que se utilizan para diagnósticos y seguimientos, por ejemplo, pueden variar considerablemente, desde aquellos basados en criterios epidemiológicos tradicionales (i. e., prevalencias), hasta indicadores sociales y económicos más amplios y aparentemente sin relación directa con la salud mental (i. e., indicadores de vivienda). La alternativa ha sido asumir una transición de la primera a la segunda perspectiva a manera de asimilación, en distintos grados, que desemboca en la integralidad del concepto y de las propuestas de intervención. Se trata de un tránsito hacia 
lo que se considera ideal, que no se corresponde necesariamente con la realidad de las personas, los colectivos y las instituciones.

En todo el mundo, el número de personas con trastornos mentales comunes (i. e., ansiedad y depresión) viene en aumento desde 1990. La OMS/Banco Mundial estima el ascenso de 416 a 615 millones de personas entre 1990 y 2013. La cifra corresponde al número de individuos que requerirían algún tipo de atención en salud mental considerada de una manera convencional. Ese sería el foco de la intervención en la primera perspectiva, la convencional, aunque en ese caso la patología tiene antecedentes sociales, los únicos que podrían explicar el incremento. El costo de no tratar estos problemas que implican una carga de enfermedad alta es significativo, como también lo es el retorno de la inversión en su tratamiento [12].

Desde la segunda perspectiva, la presión por acciones encaminadas a la promoción y mantenimiento de la salud mental sería muy superior y el tipo de enfoque tendría que cambiarse: del individuo a la sociedad; en ese sentido, se asume plenamente una mirada de salud pública. Se reconoce, por ejemplo, que las transformaciones sociales llevan un ritmo acelerado y sus efectos en las personas son cada vez más directos [13]. Estudios de cohorte muestran el incremento de la ansiedad a través de generaciones, lo que sin duda tiene relación con condiciones culturales y socioeconómicas [14]. En una definición amplia de la salud mental, en la que desempeñan un papel central los determinantes sociales, las estrategias orientadas fundamentalmente a factores individuales no tienen sentido. Aunque la opción farmacéutica ha sido la solución mejor aceptada por la sociedad, no ha logrado contrarrestar el incremento de los problemas de salud mental y habría dudas de que el futuro de la salud mental sea el desarrollo de nuevos medicamentos por parte de la industria farmacéutica.

El estigma de la enfermedad mental complica el panorama. Aun si existieran todos los recursos necesarios para brindar atención, las atribuciones socioculturales negativas que hacen parte de las formas en las que las personas entienden la salud mental constituyen una de las barreras más importantes para el acceso a la atención. Discriminación y violación de derechos hacen parte de la cotidianidad de las personas que sufren algún tipo de problema de salud mental. En todo el mundo, los encargados de la salud mental se enfrentan permanentemente a este problema, y debe ser, por lo tanto, uno de los ejes articuladores de las políticas públicas. No es infrecuente que las políticas de salud estén dislocadas de representaciones sociales que reproducen temores y estereotipos. Los diagnósticos sirven para mantener divisiones simbólicas y barreras entre normalidad y locura [15]. La falta de liderazgo y voluntad política, así como la organización del sistema de salud son otras barreras importantes.

El impacto socioeconómico de los problemas de salud mental es enorme. Los estudios sobre carga de enfermedad muestran el costo de los trastornos mentales en términos de discapacidad y muerte prematura [16]. Desde la primera perspectiva, la convencional centrada en la patología, la carga de enfermedad se visibiliza en condiciones como el trastorno depresivo. En la segunda perspectiva, la extendida y centrada en el bienestar, en causas externas tales como accidentes de transporte. Ambas causas se encuentran entre los cinco primeros lugares de carga de enfermedad. Si el costo de los problemas de salud mental no tratados es superior al de su atención, se debe en parte a la discapacidad que producen.

Los problemas de salud mental además no tienen causas específicas reconocidas. Los más costosos para la sociedad, como los trastornos del espectro autista o la esquizofrenia, para dar ejemplos, no tienen una causa única o forma de manejo que conduzcan a la curación. Las cargas de enfermedad más altas dentro de los trastornos mentales y del comportamiento corresponden al trastorno depresivo mayor, los trastornos de ansiedad, el uso de sustancias psicoactivas y la esquizofrenia [17]. Los trastornos de ansiedad con frecuencia ingresan al sistema de salud por los servicios de urgencias o la consulta de medicina general. Usualmente, pasan desapercibidos como problemas de salud mental.

Los trastornos mentales tienen también una importante variabilidad histórica; sus conceptualizaciones se transforman en el tiempo. En la primera edición del DSM (1952), por ejemplo, las adicciones eran una categoría del trastorno de personalidad sociopática. Esta categoría ha tenido transformaciones en las ediciones subsiguientes. Los términos aparecen y desaparecen, y algunas entidades cambian de categoría (i. e., juego 
patológico, en el DSM 5 aparece en la categoría de trastornos adictivos). Con frecuencia, estos cambios van ligados a transformaciones sociopolíticas que se presentan como nuevos hallazgos. Dentro de la serie del DSM, ninguna edición había sido tan cuestionada como la 5. a. Los trastornos disfórico-premenstrual, neurocognitivo leve, la disforia de género, la desregulación disruptiva del ánimo, y los del espectro autista, para mencionar algunos, hacen parte de la gama de trastornos mentales problemáticos y suscitan intensa controversia [18].

Las condiciones sociales son el determinante más importante de la salud mental, tanto en su origen como en el proceso de atención. Los ejemplos abundan, aun para los trastornos mentales más graves, por ejemplo, los refugiados tienen un riesgo mayor de psicosis en comparación con la población general y otros migrantes no refugiados [19]. Se debe resaltar dentro del conjunto de dificultades con las que tropieza la formulación e implementación de una política de salud mental además de las conceptuales, las características del sistema de salud. Una política con énfasis social puede ser irreconciliable con un sistema de salud basado en el mercado.

\section{Antecedentes planteados en Colombia hasta 1993 en torno a la formulación de políticas de salud mental}

En conjunto, todos los aspectos mencionados hacen ardua y compleja la tarea de formular e implementar políticas nacionales de salud mental. En el caso de Colombia, hay una historia de las transformaciones en la conceptualización de la salud mental y de los intentos de desarrollar e implementar políticas de salud mental que parece reproducirse en el tiempo. Circunstancias de diverso orden, incluyendo por supuesto y principalmente sociopolíticas, han transformado los modos de concebir e interpretar el fenómeno, y en consecuencia las respuestas sociales e institucionales para abordarlo.

Aunque en 1960 se creó la Sección de Higiene de Salud Mental en el Ministerio de Salud Pública [20], transformada en 1963 a Sección de Salud Mental, sin que la de Higiene hubiera empezado a funcionar ni tuviera recurso humano o presupuesto, fue a comienzos de la década de los setenta cuando el tema de la salud mental comenzó a visibilizarse, tímidamente, en la agenda de la salud pública [21].

EL Sistema Nacional de Salud de los setenta, fragmentado, de oferta escasa, y en el que el acceso a la prestación de servicios dependía de la capacidad económica de la persona, se reformó en 1990 (Ley 10), estructurándose alrededor de principios que manifiestan valores de la sociedad, entre ellos derechos humanos y participación.

El macrodiagnóstico de salud mental de 1973 es un referente histórico, por cuanto en esos momentos se inició el proceso de liberación de la salud mental de su proscripción de los temas de salud, estando anteriormente relegada a la institución del asilo [22]. De hecho, la división de salud mental del Ministerio de Salud fue creada en 1974. Con los macrodiagnósticos de salud mental de 1981 y de 1986-1987 se hizo una descripción del sector en términos de capacidad instalada y otros recursos disponibles, considerando además el refuerzo de alternativas de atención diferentes al hospital psiquiátrico [23,24] . La salud mental se entendía en ese entonces desde la postura convencional de la distinción entre normalidad y anormalidad.

Un giro a concepciones más amplias se empezó a consolidar en la década de los noventa, en el contexto de profundas reformas neoliberales del Estado, privatizaciones y guerra contra las drogas. En 1990 se creó la División de Comportamiento Humano en el Ministerio de Salud; el cambio de nombre reflejaba transformaciones en la forma de conceptualizar la salud mental. La Ley 10 de 1990 que reorganizó el Sistema Nacional de Salud fue el marco general de esos cambios [25]. A partir de una serie de principios, entre los que podemos destacar la participación social, se comenzó a orientar el enfoque a perspectivas ampliadas de salud.

En 1993 se realizó el Primer Estudio Nacional de Salud Mental [26], una investigación que incluía indicadores epidemiológicos tradicionales, por ejemplo, prevalencias, para un conjunto de trastornos. En ese caso el concepto comenzó a ampliarse, pero el estudio de la problemática permanecía atado a enfoques 
convencionales, estableciéndose uno y otros en tiempos distintos. Se empieza a poner entonces en evidencia que la conceptualización de la salud mental avanza a un ritmo distinto al de la transformación de las prácticas, lo que va a caracterizar un primer factor de disociación con implicaciones en la formulación e implementación de políticas.

Pero en 1993 se creó el Sistema de Seguridad Social Integral, que implicó una reforma de mercado para el sector salud [27]. En esa lógica del mercado, la prestación de servicios de salud mental se cuestiona desde la viabilidad financiera. De ese modo, las pretensiones sociales chocan con la lógica de la economía de mercado; los gérmenes de nuevas conceptualizaciones de la salud mental se enfrentaron con las condiciones concretas de funcionamiento del sistema de salud, lo que provocó un segundo factor de disociación entre conceptos y prácticas. Si bien el sistema de salud ha sido capaz de incrementar considerablemente la cobertura, dicha cobertura es ilusiva o al menos imposible de cumplir de acuerdo con las expectativas de la sociedad. El resultado es la creación de una gran cantidad de barreras de acceso de diversa índole, entre ellas la falta de una perspectiva de salud pública en salud mental.

\section{Antecedentes del caso colombiano entre 1994 y 2013}

En términos de conceptualización de la salud mental, la ausencia de enfermedad parece ser actualmente la perspectiva favorita, y es la que prevalecido en el tiempo desde 1993 [28]. En los últimos años, sin embargo, los enfoques positivos de salud mental se han ido integrando con los centrados en la patología. En Colombia, en términos formales, tanto en los documentos técnicos producidos como en la legislación, se optó por una definición positiva de salud mental acorde con tendencias internacionales [29].

Con el paso del tiempo, los determinantes sociales han comenzado a ocupar también un lugar importante en la agenda de la salud mental [30]. Dentro del conjunto de determinantes, las relaciones sociales se destacan como factor fundamental. La historia a la que hacemos referencia es la que va de la preeminencia de una perspectiva convencional centrada en la patología a los brotes, en esta nueva perspectiva, de un enfoque de determinantes sociales en los discursos sobre políticas públicas.

Luego del Estudio de Salud Mental de 1993, en Colombia se han desarrollado varios intentos de formulación cuya recepción por parte de la sociedad y sus instituciones ha sido fútil y es evidente la falta de liderazgo. Se debe reconocer, sin embargo, las contribuciones de tales intentos a visibilizar a la salud mental y su problemática a lo largo de los últimos veinte años. La Política Nacional de Salud Mental para Colombia fue formulada en 1998 [31], con intentos de reformulación en 2005 y 2007 bajo la coordinación de la Fundación FES Social y la Asociación Colombiana de Psiquiatría, respectivamente [32,33]. La Ley 1616 de 2013 señaló la inminente necesidad de un nuevo ajuste efectivo a la política de 1998, aún vigente [28]. En ese marco, se desarrolló la iniciativa de ajuste del 2014, que es objeto central de esta reflexión; no obstante, en el 2015 y luego de trazados los citados lineamientos de ajuste de 2014, el Ministerio de Salud dio a conocer un nuevo estudio de salud mental colombiano, la Encuesta Nacional de Salud Mental Colombia 2015 [34].

\section{El estudio de salud mental de 1997}

En 1997 se realizó un segundo estudio nacional de salud mental, también desde una caracterización epidemiológica reducida, en el que no se utilizaron los mismos criterios del estudio anterior, lo que dificultó el ejercicio de comparaciones. No fue publicado oficialmente, aunque sus resultados parciales y preliminares fueron dados a conocer al público por diferentes medios [35,36]. La falta de una tradición acumulativa de información y logros se traduce en la sensación de que cada desarrollo marca un nuevo comienzo. 


\section{La politica nacional de salud mental de 1998}

Distintas circunstancias presionaron al Estado para formular una política nacional de salud mental, entre ellas, el despropósito que implica negar la relevancia de la salud mental en la salud, intensificación del conflicto armado, reformas globales en la atención psiquiátrica que hacían parte de compromisos adquiridos por Colombia [37], preocupación por el incremento en las manifestaciones de violencia cotidiana, derechos humanos, entre otros. El conjunto de factores condujo a la formulación de lineamientos para elaborar una política nacional de salud mental, prevención de la violencia y promoción de la coexistencia pacífica por parte de la División de Comportamiento Humano del Ministerio de Salud, que fue luego sometida a la participación y concertación con un buen número de agentes gubernamentales y representantes de organizaciones sociales para producir la primera política nacional de salud mental para Colombia (PNSM). La política fue adoptada mediante la Resolución 2358 de 1998, a escasos veinticinco días de que finalizara el gobierno de Ernesto Samper Pizano (1994-1998), en medio de un complejo entorno nacional a raíz de los escándalos de financiación de su campaña a la presidencia y del inicio del gobierno de Andrés Pastrana Arango (1998-2002), el cual quería desmarcarse drásticamente de su predecesor, lo que a fin de cuentas influyó en la precaria implementación de esa política.

La PNSM se fundamentó en una definición positiva de salud mental, enfatizando capacidades, potencialidades, bienestar subjetivo y logro de metas. Esta tendencia va a continuar en los documentos técnicos nacionales que posteriormente serán discutidos.

Ante las dificultades en la implementación de la PNSM, de acuerdo con las características del Sistema General de Seguridad Social en Salud (SGSSS), por lo que aquí hemos denominado factores de disociación, se buscó salida en la preparación de nuevos lineamientos que, fundamentados en las mismas conceptualizaciones de la PNSM, pudieran concretar las aspiraciones de la política en modelos de atención y prestación de servicios. Se marcó así una nueva división entre actores de la política: los de una perspectiva técnica y de manejo gerencial, la cual pretendía resolver el problema desde el lenguaje de la racionalidad económica, y los de una perspectiva más social que para quienes se ubicaban en la primera posición, postulaban ilusiones; Homo economicus versus Homo moralis.

\section{El Estudio Nacional de Salud Mental colombiano de 2003}

El Estudio Nacional de Salud Mental Colombia 2003 [26] continuó en la perspectiva de estudios epidemiológicos con indicadores tradicionales y según criterios diagnósticos del DSM. Incluyó además medidas de asociación, específicamente razones de disparidad. Mostró que los trastornos de ansiedad son los de mayor prevalencia en el país, en contraste con los estudios de 1993 y 1997 que ubicaban la mayor prevalencia en la depresión. Los estudios de todas maneras no son comparables.

\section{Los lineamientos de ajuste a la Politica Nacional de Salud Mental de 2005 y 2007}

La perspectiva de la salud mental positiva, inaugurada por el Estudio Nacional de 1993, fue retomada por los documentos técnicos de 2005, Lineamientos de políticas de salud mental para Colombia, y de 2007, Politica nacional del campo de la salud mental, ambos intentos fallidos de realizar nuevas formulaciones de política $[23,24]$.

No obstante, en las conceptualizaciones, especialmente del segundo, se empezó a considerar el papel de los determinantes sociales, la perspectiva de derechos, se señalaron prioridades y se establecieron líneas de acción y estrategias. 
El análisis de ambos documentos muestra que, si bien ambos comparten temáticas y contenidos, también existen diferencias no fundamentales que sí señalan el efecto de la diferencia de contextos. El documento de 2005 sería más gerencial, mientras que el del 2007 fue más social. Al final, ni siquiera los defensores de la racionalidad microeconómica lograron proponer una solución a la cuestión de la implementación de políticas, por ejemplo, cómo traducir expectativas en promoción, prevención y prestación de servicios de acuerdo con las características del Sistema General de Seguridad Social en Salud (SGSSS).

\section{Experiencia 2014: un ajuste a la política pública de salud mental colombiana}

La ley 1616 de 2013, o Ley de Salud Mental, decretó el pleno derecho a la salud mental en los componentes de promoción, prevención y atención, fundamentándose en los principios de la atención primaria en salud. Al mismo tiempo, ordenó el ajuste de la PNSM de 1998 y su implementación, de acuerdo con los criterios especificados por la ley. Los criterios fueron: a) reformular la política desde una definición positiva de salud mental; b) centrada en derechos; c) siguiendo estrategias de atención primaria en salud; d) con énfasis en participación; ye) considerando acciones intra e intersectoriales para el abordaje de los determinantes sociales de la salud mental [28].

Con base en esas directrices y seleccionados por el Ministerio de Salud y Protección Social como equipo consultor para la reformulación de la PNSM, procedimos como etapa inicial a realizar un análisis de los contenidos de la PNSM de 1998 y de los documentos sobre propuestas de ajuste de 2005 y 2007. Tratamos de recopilar con el análisis la experiencia acumulada durante quince años de trabajo en políticas de salud mental. El análisis se complementó con información epidemiológica disponible hasta ese momento, revisión del estado de la investigación sobre políticas públicas de salud mental en el mundo y la elaboración de listados de organizaciones sociales y gremiales, y de agencias estatales, con el fin de discutir los principios sobre los que se estructuraría la política.

La elección de organizaciones sociales para incluir evidenció el espíritu del equipo consultor, en cuanto a facilitar la participación directa en la propuesta de ajuste a grupos humanos vulnerables y habitualmente excluidos de este tipo de ejercicios, pero en cambio sujetos habitualmente a la aplicación de una política de salud mental, entre los que se contaron: personas con discapacidad mental-psicosocial organizadas; organizaciones de familiares de personas con discapacidad mental-psicosocial y cognitiva incluyendo FNNA (familiares de niños, niñas y adolescentes con dicha condición); personas pertenecientes a grupos de víctimas de violencia por conflicto armado; grupos organizados de la comunidad LGBTI (lesbianas, gays, bisexuales, transgeneristas, e intersexuales); organizaciones que velan por la salud de las mujeres; organizaciones y colectivos que velan por los derechos humanos en general y de las personas con discapacidad en particular; organizaciones de indígenas, afrodescendientes y palenqueros, entre otras convocadas. Igualmente, fue amplia la convocatoria de organizaciones gremiales, incluyendo gremios de psiquiatras, psicólogos, trabajadores sociales y enfermeras, así como organizaciones académicas, entre otros. Entre los agentes estatales, se convocaron aquellos pertenecientes al sector salud nacional, regional y local, ligados al sector de reintegración social y de bienestar, entre otros.

De acuerdo con los antecedentes expuestos, la PNSM 2014 se fundamentó en una definición positiva de salud mental, es decir, en capacidades, logros y contribuciones del individuo. Existen diversas perspectivas desde las que se conceptualiza la salud mental, entre ellas se encuentra la de la salud mental positiva que enfatiza virtudes humanas y fortalezas del carácter, por lo que puede considerarse una perspectiva moral de la salud mental [38]. En esa perspectiva, el énfasis no es el tratamiento médico de la patología, sino la promoción de capacidades y el desarrollo del potencial humano. Dicha conceptualización de la salud mental ha estado en boga en los últimos años y se ha extendido en forma paralela al crecimiento de la psicología positiva [39].

El problema que presenta la perspectiva positiva, en términos de la formulación de una política de salud mental, es el riesgo de convertirse en un ideal imposible de implementar. Pero una perspectiva positiva de 
la salud mental ofrece la ventaja de la congruencia con valores culturales, de forma tal que una política con ese enfoque traduciría adecuadamente las expectativas del grupo al que sirve. Asimismo, permitiría intervenir sobre el sistema de relaciones sociales para producir resultados en indicadores como integración social, contribución al grupo, confianza social, tolerancia, relaciones positivas con otros y bienestar subjetivo, todos relacionados con la salud mental.

El temor de formular una política desde tal perspectiva radicaría en las cargas que supone para el sistema de salud y en los interrogantes sobre la validez empírica de las intervenciones. En todo caso, incluso una definición positiva de salud mental debe acogerse a especificidades culturales, sin asumir por lo tanto la universalidad de sus supuestos [40]. La Ley 1616 de 2013 estableció como prioridad de la PNSM la perspectiva de salud mental positiva; la salud mental pasó a ser definida en esos términos [28].

Previa identificación de las problemáticas de salud mental con enfoque diferencial, a través de cinco categorías distintas, relacionadas con determinantes sociales de la salud, por ejemplo, clase social, género, etnia, discapacidad, y curso de la vida, se estableció una caracterización de la situación de salud mental de los colombianos. La visión propuesta fue "A 2021 el país logra el goce efectivo del derecho a la salud mental mediante su posicionamiento en la agenda pública y la respuesta social organizada desde diferentes sectores, bajo la responsabilidad indelegable del estado". A partir de un valor único que se consideró fundamental, el de la ciudadanía, se discutieron y consignaron nueve principios que orientarían las áreas de actuación. Los principios contemplados en la PNSM 2014 fueron los de inclusión, participación, calidad, equidad, integralidad, universalidad, corresponsabilidad, intersectorialidad y accesibilidad.

Desde los principios establecidos se definieron ocho áreas de actuación: promoción, prevención, atención integral, rehabilitación, respuesta social, gestión de conocimiento, talento humano y dirección. Las áreas de actuación no son una innovación de la PNSM 2014, por el contrario, recogen trabajos y discusiones previas, integrándolos, con el fin de asegurar que la política recupera trabajos anteriores realizados por el Ministerio de Salud para construir una política sinérgica que tenga en cuenta la pluralidad de perspectivas sobre salud mental. De eso se trata una política pública.

Otro aspecto que retomó la PNSM 2014, siguiendo las indicaciones de la Ley 1616, fue el del papel de los determinantes sociales. La política fue explícita en asumir que la salud mental es muy sensible a factores sociales, que los orígenes de malestar están ligados fundamentalmente a las condiciones de vida de la población, y por lo tanto las intervenciones y la prestación de servicios deben basarse en esa premisa en primer lugar. Gradiente social, empleo y género son asuntos clave que considerar. Los trastornos mentales tienen un patrón social definido que por lo general incluye variables como nivel de ingreso, género, educación y estado civil [41].

Los resultados de la consulta ciudadana para formular el Plan Decenal de Salud Pública 2012-2021 mostraron como áreas prioritarias para la sociedad el consumo de sustancias psicoactivas y las violencias, incluidas accidentalidad y suicidios. Específicamente, la dimensión Convivencia Social y Salud Mental incluyó entre sus componentes la promoción de la salud mental y la convivencia, y la prevención y atención integral a problemas y trastornos mentales. Estos aspectos fueron tenidos en cuenta en el proceso de formulación, que resaltó la promoción de la convivencia pacífica y la prevención de la violencia como acciones integradas a las estrategias de acción propuestas.

El trabajo de análisis de los documentos previos, el estudio del estado de la investigación, y la caracterización epidemiológica sirvieron de base para proponer los temas y componentes de la PNSM en tres rondas consecutivas de discusión con actores gubernamentales y organizaciones sociales. Además de las dependencias correspondientes del Ministerio de Salud y Protección Social, participaron los referentes de salud mental de los departamentos, las secretarías de salud de las principales ciudades del país, asociaciones gremiales de enfermería, neurología, psicología, psiquiatría, trabajo social, y diferentes organizaciones sociales de personas con discapacidad cognitiva y mental, víctimas de violencia, diversidad sexual, y minorías étnicas, como se ha reseñado en secciones anteriores. En suma, participaron 27 organizaciones de la sociedad civil, así como 
35 referentes de salud mental departamentales y municipales, coordinados por el Ministerio de Salud y Protección Social con el apoyo de la Organización Panamericana de la Salud.

Existen diversas metodologías para asegurar la participación pública en procesos de formulación de políticas, cada uno con sus propias ventajas y desventajas. Los temas que surgen son los de cómo informar a la ciudadanía sobre el proceso y cómo lograr su participación. Una primera aproximación a las necesidades y preferencias de los ciudadanos la ofrecieron desarrollos previos como el del Plan Decenal de Salud Pública, los estudios de salud mental, estado de la investigación sobre el tema y documentos anteriores a la PNSM 2014, incluyendo políticas regionales de salud mental.

En el caso de la consulta y participación en la formulación de la PNSM 2014, se tuvo en cuenta que la participación ciudadana es vital en la refrendación de políticas públicas. Se corre el riesgo, sin embargo, de volverlo un proceso muy costoso, incoherente, que al final puede resultar en una amalgama de buenas intenciones sin posibilidades prácticas de implementarse. Adicionalmente, las restricciones presupuestales y los cronogramas establecidos hacían imposible la participación ciudadana directa como forma de participación pública; el tiempo disponible para la concertación de la política fue de apenas tres meses.

Para aliviar esto, se reconocieron distintos niveles de participación ciudadana [43]. La participación ciudadana directa y la de organizaciones intermedias ocupan las escalas superiores de la tipología de Arnstein [42]. Con el fin de deliberar, participar, involucrar y establecer alianzas, se buscó generar discusiones con representantes de organizaciones sociales e instituciones estatales. Las metodologías utilizadas fueron la publicación de consideraciones generales sobre la política en páginas web y tres talleres con actores sociales, llevados a cabo en Girardot y Cartagena. La organización de talleres fue efectiva para alimentar las discusiones, obtener retroalimentación y promover compromisos. Igualmente, se habilitó durante todo el proceso un correo electrónico manejado por el equipo consultor para intercambiar información entre sesiones de trabajo con los actores participantes. Se envió una versión del documento final de consultoría a cada una de las organizaciones y entidades participantes, con el fin de retroalimentar su aporte y participación.

\section{Hechos subsiguientes: Estudio Nacional de Salud Mental Colombia 2015 y propuesta de ajuste a la Política Nacional de Salud Mental 2016}

En el 2015, y después de concluido el ejercicio de trazado de ajustes para la política de salud mental en 2014 (PNMS 2014), se publicó un nuevo estudio nacional de salud mental, en el que ya se evidencia la necesidad de ir cerrando la brecha entre conceptualización y prácticas concretas, en ese caso las de la investigación [34]. El nuevo estudio, además de aportar información sobre la prevalencia de patologías en la población, incluyó indicadores de bienestar-malestar que expresan la transformación en la conceptualización de la salud mental, el paso del enfoque en la patología a una versión extendida de bienestar socioemocional.

Los nuevos indicadores se enmarcaron en una perspectiva de salud mental positiva e incluyeron la percepción subjetiva de bienestar, felicidad, satisfacción en las relaciones sociales, empatía, percepción de apoyo social, confianza e integración social, entre otros. En cuanto a indicadores de patología, la prevalencia más alta de trastorno mental la mostró el trastorno depresivo mayor. Esos resultados son distintos a los mostrados por el estudio anterior y merecen un análisis cuidadoso.

Sobre esta base, En el 2016 se dio inicio a un nuevo proceso de formulación de una PNSM, cuyo transcurrir e impactos están aún por ser descritos y analizados. 


\section{Discusión y conclusiones}

El vacío de una política que no ha logrado implementarse tiene diversos antecedentes: a) las características propias del sistema de salud. Las políticas públicas expresan valores, creencias y aspiraciones de una sociedad. Un valor fundamental de la sociedad colombiana es la defensa de los derechos individuales; la salud se entiende como un derecho fundamental. El reconocimiento de derechos se enfrenta con las limitaciones financieras del sistema, por lo que políticas integrales e inclusivas chocan con las barreras que interponen la escasez de recursos y la racionalidad en su manejo. b) La confusión entre políticas, planes y programas de salud. De acuerdo con la Organización Mundial de la Salud [37,44], una política es un conjunto de valores y principios. Las políticas se concretan en un plan, que, a través de estrategias, desarrolla los objetivos de la política. Un programa es una intervención específica. c) La falta de articulación entre lo nacional y lo regional. Fallas en la comunicación pública y en la difusión de la política tienen entre las consecuencias en que se formule una política nacional desconocida en las regiones, las que a su vez formulan sus propias políticas, sin tener en cuenta la política nacional, la cual queda inoperativa. d) El individualismo como ideología dominante que conduce a la preferencia por tipos de prácticas e intervenciones. El individualismo es la doctrina que sustenta a la democracia, al liberalismo y al capitalismo; establece al individuo como agente de la acción y determina las explicaciones sobre el comportamiento. En esa doctrina tienen preferencia las explicaciones individuales de los trastornos mentales. e) Las aspiraciones y expectativas de la política, que traducen las expectativas de los ciudadanos que participan en la formulación, pueden ser consideradas por los tomadores de decisión imposibles de cumplir.

La PNSM 2014 se encuentra articulada por una definición positiva de salud mental. Asume una perspectiva integral, centrada en derechos, enmarcada en la APS y orientada por la colaboración intersectorial; por tanto, acoge las indicaciones de la Ley 1616 de 2013. De ahí que los principios que la sustentan se desprenden de una conceptualización extendida de la salud mental y denotan las transformaciones de los últimos cuarenta años en la forma de entenderla. Esto se cuenta entre sus principales logros de formulación.

En general, una política pública debería estar orientada a promover el bienestar de la sociedad, prevenir daños, proteger mediante atención adecuada y considerar las diferencias en perspectivas, propio de una democracia.

El contexto sociopolítico de Colombia demanda la implementación de la PNSM. La violencia sociopolítica, cotidiana, de género y específicamente la intrafamiliar sigue siendo una prioridad. A la historia de exclusión que dio origen a la violencia en Colombia, se añaden los ciclos de retaliación que la reproducen, las altas expectativas de movilidad social ascendente que vuelve las relaciones sociales competitivas y violentas, y la intolerancia que produce la deshumanización.

Entre las consecuencias cabe esperar igualmente un aumento importante en eventos relacionados, como la accidentalidad vehicular, incluidas motocicletas y bicicletas, además de otros impactos más clásicamente descritos en términos de padecimientos y trastornos específicamente encuadrados dentro del campo de la salud-enfermedad mental, junto con los impactos sobre las maneras en que se organizan o alteran vínculos sociales en poblaciones afectadas por violencia sociopolítica, siendo este el probable mecanismo más importante de la traducción de la escala socioestructural a la individual.

Ninguno de los estudios de salud mental realizados hasta el momento ha incluido de manera central un análisis del impacto de la violencia política en Colombia ni de las repercusiones que tendría para la sociedad y para el sistema de salud una salida negociada a los conflictos armados del país en lo referente específicamente a la salud mental.

El estudio de salud mental de 2015, sin embargo, sí incluyó el análisis de la violencia como fuente de malestar emocional, destacando el efecto de la violencia intrafamiliar, además de datos sobre desplazamiento forzado y exposición a diversos eventos relacionados con el conflicto armado [34]. Una de las principales 
limitaciones para la realización de la experiencia de ajuste PNSM 2014 fue la ausencia de este renovado estudio de la salud mental colombiana, para el momento de inicio, realización y conclusión de lo propuesto.

En las condiciones de alteración del tejido social que se observan en Colombia, la violencia cotidiana deberá ser un foco de intervenciones desde planes y programas. La PNSM 2014 fue un proceso que consideró los aspectos mencionados en este artículo para reconsiderar la PNSM 1998 de acuerdo con las características de un país transformado y en situación de cambio acelerado, siendo este otro de sus logros. Corresponde al gobierno definir si esa política reformulada se implementa, si se retomará por los ejercicios posteriores emprendidos, o si buscarán nuevas formulaciones para reproducir la historia de la no implementación, a riesgo de inconsistencia y falta de compromiso.

En el 2016 se dio inicio a un nuevo proceso de formulación de una PNSM, teniendo en cuenta los resultados del estudio de 2015 y el nuevo modelo de atención en salud. En cualquier caso, una PNSM no se puede someter forzosamente a las características de organización del sistema de salud porque, aunque original, formular una política al revés no deja de ser problemático.

Está pendiente la descripción e investigación sobre los procesos vividos durante el nuevo intento de actualización de la PNSM en 2016, así como la elaboración de balances sobre los logros que con ella se consigan en favor de la salud mental, de cara no solamente a las particularidades señaladas por el último estudio nacional de salud mental, incluyendo no solo reflexiones específicas sobre la violencia en sus distintas manifestaciones, problema que en general es difícil de estimar, aunándose a ello que los pocos estudios realizados a la fecha arrojan resultados disímiles [31], sino además afrontando los retos de escenarios posteriores a los acuerdos.

Los mecanismos que actúan en cómo procesos sociales, en conjunto con otros tantos característicos de la estructura social colombiana, afectan la salud mental de los individuos y los colectivos humanos, hacen parte de programas de investigación cuyo desarrollo reclama la mayor atención.

\section{Referencias bibliográficas}

1. Mnookin S. Out of the shadows: Making mental health a global development priority. Washington D. C.: World Bank Group; 2016.

2. World Health Organization. Mental health: strengthening our response (Fact sheet No. 220). Ginebra: World Health Organization; 2010.

3. Organización Panamericana de la Salud. Acerca de OPS Colombia: OPS/OMS; 2017.

4. José A. De compromisos internacionales a políticas públicas. La viabilidad de la Agenda 2030. Madrid: CIDOB. Colección Monografías; 2017.

5. Roth AN. Políticas públicas. Formulación, implementación y evaluación. Bogotá: Ediciones Aurora; 2002.

6. Patel V, Kleinman A. Poverty and common mental disorders in developing countries. Bulletin of the World Health Organization. 2003; 81(8):609-15.

7. Perilla M, Cadena L. Formación del discurso en salud mental en la primera mitad del siglo XX. MedUnab. 2014; 16(3):118-26.

8. Henao S, Quintero S, Echeverry J, Hernández J, Rivera E, López S. Políticas públicas vigentes de salud mental en Suramérica: un estado del arte. Revista Facultad Nacional de Salud Pública. 2016; 34(2):184-92.

9. Restrepo-Zea E. El Hospital San Juan de Dios 1635 1895. Una historia de la enfermedad, pobreza y muerte en Bogotá. Bogotá: Universidad Nacional de Colombia. Facultad de Ciencias Humanas. Centro de Estudios Sociales; 2011.

10. González L, Peñaloza R, Matallana M, Gil F, Gómez-Restrepo C, Vega A. Factores que determinan el acceso a servicios de salud mental de la población adulta en Colombia. Revista Colombiana de Psiquiatría. 2016; 45(S1):89-95. 
11. Molina C. El campo de la salud mental y la política pública como espacio de gestión participativa de la animación sociocultural. ATPS. 2016; (11):43-50.

12. Chisholm D, Sweent K, Sheehan P, Rasmussen B, Smit F, Cuijpers P, et al. Scaling-up treatment of depression and anxiety: a global return on investment analysis. The Lancet Psychiatry. 2016; 3(5):415-24.

13. Giddens A. Un mundo desbocado. Los efectos de la globalización en nuestras vidas. Bogotá: Taurus; 2000.

14. Twenge JM. The age of anxiety? Birth cohort change in anxiety and neuroticism, 1952-1993. Journal of Personality and Social Psychology. 2000; 79(6):1007-21.

15. Jodelet D. Madness and social representations. Living with the mad in one French community. Berkeley: University of California Press; 1991.

16. Murray CJL, Lopez AD, editors. The global burden of disease. A comprehensive assessment of mortality and disability from diseases, injuries, and risk factors in 1990 and projected to 2020. Boston, MA: Harvard University Press; 1996.

17. Murray CJL, et al. Disability-adjusted life years (DALYs) for 291 diseases and injuries in 21 regions, 1990-2010: a systematic analysis for the Global Burden of Disease Study 2010. Lancet. 2012; 380(9859):2197-23.

18. Sandín B. DSM 5: ¿Cambio de paradigma en la clasificación de los trastornos mentales? Revista de Psicopatología y Psicología Clínica. 2013; 18(3):255-86.

19. Hollander A-C, Dal H, Lewis G, Magnusson C, Kirkbride JB, Dalman C. Refugee migration and risk of schizophrenia and other non-affective psychoses: cohort study of 1.3 million people in Sweden. BMJ. 2016; 352:11030.

20. Decreto número 1423 de 1960. Por el cual se reorganiza el Ministerio de Salud Pública y se le adscriben los negocios de que debe conocer.

21. Decreto 3224 de 1963. Por el cual se reorganiza el Ministerio de Salud Pública y se adscriben los negocios que debe conocer.

22. Ministerio de Salud. Macrodiagnóstico de salud mental, 1973. Bogotá: Ministerio de Salud; 1974.

23. Ministerio de Salud. Macrodiagnóstico de salud mental. Subsector oficial directo. Bogotá: Ministerio de Salud; 1982.

24. Ministerio de Salud, República de Colombia. Macrodiagnóstico de salud mental. Subsectores oficial directo y privado 1986-1987. Bogotá: Ministerio de Salud; 1988.

25. Ley 10 de 1990. Por la cual se reorganiza el Sistema Nacional de Salud y se dictan otras disposiciones.

26. Ministerio de Salud. Estudio Nacional de Salud Mental y Sustancias Psicoactivas Colombia 1993. Bogotá: Ministerio de Salud; 1994.

27. Ley 100 de 1993. Por la cual se crea el Sistema de Seguridad Social Integral y se dictan otras disposiciones.

28. Ley 1616 de 2013. Por medio de la cual se expide la ley de salud mental y se dictan otras disposiciones.

29. Urrego-Mendoza Z. Reflexiones en torno al análisis de la situación de salud mental en Colombia 1974-2004. Revista Colombiana de Psiquiatría. 2007; 36(2):307-19.

30. Herrman H, Saxena S, Moodie R, editors. Promoting mental health. Concepts, emerging evidence, practice. Ginebra: World Health Organization; 2005.

31. Resolución 2358 de 1998. Política Nacional de Salud Mental.

32. Ardón-Centeno N, Cubillos-Novella A. La salud mental: una mirada desde su evolución en la normatividad colombiana. 1960-2012. Revista Gerencia y Políticas de Salud. 2012; 11(23):12-38.

33. Comité de Políticas de la Asociación Colombiana de Psiquiatría. Editorial. La Política Nacional del Campo de la Salud Mental: entre el escepticismo y la esperanza. Revista Colombiana de Psiquiatría. 2008; 37(3):302-3.

34. Minsalud, Colciencias. Encuesta Nacional de Salud Mental 2015.

35. Torres-DeGalvis Y, Montoya I. Segundo estudio Nacional de Salud Mental y Consumo de Sustancias Psicoactivas - Colombia 1997. Bogotá: Fundación Universitaria Luis Amigó; 1997. 
36. Torres-De-Gálvis Y. Epidemiología de los trastornos psiquiátricos - Estudio Nacional Colombia. Psiquiatríacom [internet]. 1999 [acceso: 17 de sepriembre de 2017]; 3(4). Disponible en: https://psiquiatria.com/trabajos/49 3.pdf

37. Organización Panamericana de la Salud, editor. Reestructuración de la atención psiquiátrica: bases conceptuales y guías para su implementación. Memorias de la Conferencia Regional para la Reestructuración de la Atención Psiquiátrica; 1990; Caracas, Venezuela. Washington, D.C.: OPS; 1991.

38. Vaillant GE. Mental health. American Journal of Psychiatry. 2003; 160(8):1373-84.

39. Seligman MEP, Csikszentmihaly M. Positive psychology: An introduction. American Psychologist. 2000; 55(1):5-14.

40. Vaillant GE. Positive mental health: is there a cross-cultural definition. World Psychiatry. 2012; 11(2):93-9.

41. Ross CE, Mirowsky J. Explaining the social patterns of depression: control and problem solving or support an talking. Journal of Health and Social Behavior. 1989; 30(2):206-19.

42. Arnstein SR. A ladder of citizen participation. Journal of the American Institute of Planners. 1969; 35(4):216-24.

43. Vargas-Cuellar M. Niveles de participación ciudadana en las políticas públicas: Una propuesta para el estudio de mecanismos institucionales de participación. Tlatemoani Revista de Investigación Académica. 2010; (2):1-8.

44. Organización Mundial de la Salud. Políticas, planes y programas de salud mental. Editores Médicos Edimsa; 2006.

\section{Notas}

*Artículo de reflexión

\section{Licencia Creative Commons CC BY 4.0}

Como citar este artículo: Parales-Quenza CJ, Urrego-Mendoza ZC, Herazo-Acevedo E. La insoportable levedad de la política nacional de salud mental para Colombia: reflexiones a propósito de la propuesta de ajuste en 2014. Rev Gerenc Polít Salud. 2017; 17(34): 1-14. https://doi.org/10.11144/Javeriana.rgps17-3 4.ilpn 